\title{
Myocardial Perfusion Imaging in the Evaluation of Outcome of Coronary Intervention in Coronary Artery Disease Patients
}

\author{
Sanhita Majumder ${ }^{1}$, Shamim Momtaz Ferdousi Begum ${ }^{1}$, Ankan Kumar Paul ${ }^{2}$, and Chayan Singha ${ }^{3}$

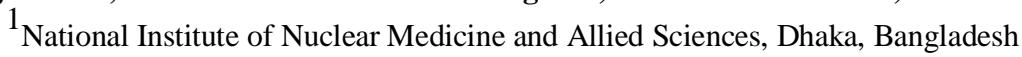 \\ 2 Department of Cardiac Surgery, National Institute of Cardiovascular Diseases, Dhaka, Bangladesh, \\ ${ }^{3}$ Department of Cardiology, Bangabandhu Sheikh Mujib Medical University, Dhaka, Bangladesh \\ Correspondence Address: Dr.Sanhita Majumder, M Phil (Nuclear Medicine), NationalInstitute of Nuclear Medicine and Allied \\ Sciences, Dhaka, Bangladesh, E-mail:sanhita136@gmail.com
}

\begin{abstract}
Objective: Coronary artery disease (CAD) is an important medical and public health issue because it is one of the leading causes of death and disability throughout the world and is rapidly emerging as a major cause of mortality in developing countries including Bangladesh. SPECT-MPI is a cost- effective and non invasive means of identifying ischemic and viable myocardium along with its vascular distribution. This study is aimed to evaluate prospectively the ability of MPI to predict the outcome of Coronary Intervention in CAD patients of Bangladesh who are referred to National Institute of Nuclear Medicine \& Allied Sciences (NINMAS) for performing MPI.

Patients and Methods: This prospective longitudinal and observational type of study was carried out in National Institute of Nuclear Medicine \& Allied Sciences (NINMAS) located at Bangabandhu Sheikh Mujib Medical University (BSMMU) campus, from July 2017 to June 2018with 12 CAD patients who were referred here. Gated SPECT MPI was performed on the selected patients before any coronary intervention to predict myocardial viability \& probable outcome of intervention. A follow up MPI of the same patients was performed after minimum 3 to 4 months of revascularization procedure to determine the outcome of intervention and to detect in stent restenosis or new onset ischemia if present which can be prevented by additional revascularization procedure. Finally pre and post intervention MPI results were analyzed by standard statistical analysis by using the Statistical Package for Social Sciences version 20.0 for Windows (SPSS Inc., Chicago, Illinois, USA). P values <0.05 was considered as statistically significant.

Results: The sensitivity of MPI for the initial evaluation and risk stratification by diagnosing perfusion defect in the enrolled $12 \mathrm{CAD}$ patients have been found to be $90 \%, 77.8 \%$ and $81.8 \%$ in case of LAD, LCX and RCA territories respectively. Whereas, the specificity and positive predictive value have been found $100 \%$ for LAD and RCA territories. About $16.67 \%$ patients were found to have restenosis in this study diagnosed by early post-intervention MPI.

It was observed that majority of the patients had perfusion defect in LAD territory. $3(25.0 \%)$ patients had fixed defect, $5(41.7 \%)$ patients had partial reversible defect and $4(33.3 \%)$ patients had complete reversible defect in pre intervention MPI. In post intervention MPI, 4(33.3\%) patients had partially reversible perfusion defect, 2(16.7\%) patients had fixed perfusion defect, 1(3.3\%) patient was found with completely reversible perfusion defect and $5(41.7 \%)$ patients had normal MPI findings. The result was found statistically significant $(P$ value $<0.05$ ) in case of partially reversible perfusion defect and normal MPI findings when before and after intervention MPI results were compared.

The difference of mean percentage of involved myocardium by fixed defect between pre and post intervention MPI was found statistically significant $(P<0.05)$ in case of LAD territory and total LV myocardium involvement.
\end{abstract}

33.3\% patients were in high risk before undergoing intervention, whereas it was found to be $25 \%$ after intervention which was calculated on the basis of their pre and post intervention Summed Stress Score (SSS) result. Conclusion: The results of this study have indicated that SPECT MPI provides significant independent information concerning the outcome of coronary intervention in CAD patients. Furthermore, early SPECT MPI after intervention successfully identified significant improvement of myocardial viability after revascularization in patients having complete or reversible perfusion defects found in pre intervention MPI along with identifying those having restenosis. It could also depict the reduction of percentage of myocardial fixed defects within 3-4 months after intervention which is also a positive outcome of coronary revascularization.

Key words: Myocardial Perfusion Imaging, Outcome of Coronary Intervention, Coronary Artery Disease patients.

Bangladesh J. Nucl. Med. Vol. 22 No. 1 January 2019 Doi: https://doi.org/10.3329/bjnm.v22i1.40500

\section{INTRODUCTION}

Coronary Artery Disease (CAD) is one of the major causes of death and disability in developed countries. Over past decade the mortality of CAD has declined however, it remains still high and responsible for about one third or more of all deaths in individuals over age 35 in United States.CAD is rapidly emerging as a serious health problem in developing countries including Bangladesh and evidence shows that it is becoming increasingly prevalent among younger people. Bangladesh is a small country with vast population (>160 million) with a limited health statistics. According to the latest WHO data published in May 2014 coronary heart disease deaths in Bangladesh reached 50,708 or $6.96 \%$ of total deaths. The age adjusted death rate is 53.53 per 100,000 of population ranks Bangladesh number 150th position in the world (World Health Rankings, May 2014). 
The reduction in coronary artery flow in CAD may be symptomatic or asymptomatic. Myocardial ischemia or infarction may develop depending on severity and the rapidity of obstruction of coronary artery. Hence, early and accurate diagnosis is crucial and proper management is mandatory to increase the survival of the patient. Among the different investigation modalities available to assess the perfusion status of myocardium, SPECT-MPI is an effective and non invasive means of identifying ischemic and viable myocardium along with its vascular distribution. MPI plays a potential role in predicting the necessity of coronary intervention and also the probable outcome of intervention. The effectiveness of MPI in the follow-up of symptomatic and asymptomatic patients with PTCA has been defined by several studies. It is a cost saving procedure and has the ability to localize ischemia noninvasively and can detect stent restenosis.

SPECT Myocardial perfusion imaging is routinely performed at NINMAS, but no prospective data or study was conducted to assess the usefulness of SPECT MPI in patients with coronary artery disease before and after revascularization. This prospective study, before and after the coronary intervention, was aimed to provide the outcome of intervention and thereby to help in making rational management plan by the referring physicians.

\section{PATIENTS AND METHODS}

This prospective longitudinal and observational type of study was carried out in National Institute of Nuclear Medicine \& Allied Sciences (NINMAS) located at Bangabandhu Sheikh Mujib Medical University (BSMMU) campus, from July 2017 to June 2018.Twelve CAD patients who were referred to NINMAS for gated SPECT MPI at various stages of clinical workup were selected for the study by purposive sampling technique. All patients underwent MPI performed with $99 \mathrm{~m}$ Tc Sestamibi at first before undergoing coronary intervention. Single day stress - rest protocol was followed, according to the established practicing protocol of NINMAS. The patients were interviewed and complete clinical profile was recorded on the proposed questionnaire. All the patients were counseled briefly about the complete procedure and the possible side effects. Informed written consent was taken from the patients. All caffeine containing beverages were stopped for at least 24 hours and patients fasted for at least 4 hours before the stress study. Stress study was done by following one of the two following methods, 1) Exercise stress by treadmill following Modified Bruce protocol, 2) Pharmacological stress using Adenosine, Dobutamine or Dipyridamol. At stress the images were acquired on the dual headed SPECT gamma camera after 45 minutes of injection of $99 \mathrm{~m} \mathrm{Tc}$ Sestamibi (10-12 $\mathrm{m} \mathrm{Ci}$ ). Post stress acquisition with gating was done. Rest images were taken on the same day one hour after the injection of radiotracer at a higher dose (25$30 \mathrm{mCi}$ ) of $99 \mathrm{~m} \mathrm{Tc}$ Sestamibi. Then the images were reconstructed and image analysis was done. Interpretation was done by two experienced nuclear medicine physicians and abnormalities were recorded, i.e: areas of perfusion defect, type of perfusion defect, SSS etc. Information also recorded from SPECT gating such as wall motion, LVEF, size of LV cavity, TID etc. The follow up MPI was performed at an interval of minimum 3-6 months after the coronary intervention procedure.

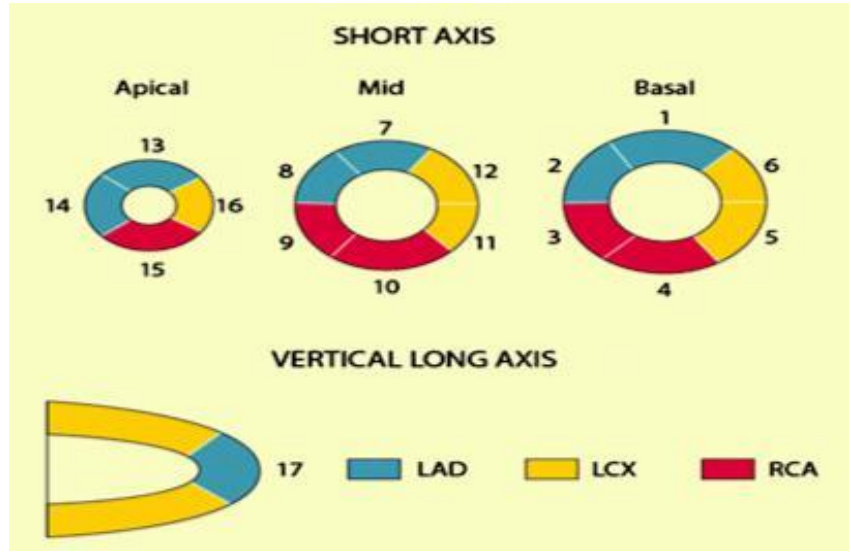

Figure 1: Standard model showing the 17 segments of the Coronary Arterial Territories corresponding to the Left Anterior Descending Artery (LAD), Right Coronary Artery (RCA) and Left Circumflex Artery (LCX). Adapted from the American Society of Nuclear Cardiology imaging guidelines for nuclear cardiology procedures: part 2,1999. Journal of Nuclear Cardiology, vol.6:, pp.G47-G48. 
Finally pre and post intervention MPI results were analyzed by standard statistical analysis by using the Statistical Package for Social Sciences version 20.0 for Windows (SPSS Inc., Chicago, Illinois, USA). Paired t-test and Z-test were used for continuous variables. Chi-square test and Mc Nemer test were used to compare categorical data. $\mathrm{P}$ values $<0.05$ was considered as statistically significant.

\section{RESULTS}

The mean age of study patients was $55.58 \pm 11.14$ (mean $\pm \mathrm{SD}$ ) years. Among 12 participants $9(75 \%)$ were male and 3 (25\%) were female; data are shown in Table-1 and Table-2. Distribution of study patients by clinical complain is summarized in Table- 3 .

Table 1: Distribution of the study subjects by age $(n=12)$

\begin{tabular}{lcc}
\hline Age (in years) & Number of patients & Percentage \\
\hline $\mathbf{4 0 - 5 0}$ & 4 & 33.3 \\
$\mathbf{5 1 - 6 0}$ & 4 & 33.3 \\
$>$ 60 & 4 & 33.3 \\
Mean \pm SD & 55.58 & \pm 11.14 \\
Range(min-max) & 40 & -79 \\
\hline
\end{tabular}

Table 2: Distribution of the study subjects by sex $(n=12)$

\begin{tabular}{lcc}
\hline Sex & Number & Percentage \\
\hline Male & 9 & 75.0 \\
Female & 3 & 25.0 \\
\hline
\end{tabular}

Table 3: Distribution of the study subjects by clinical complain $(n=12)$

\begin{tabular}{|c|c|c|c|}
\hline & \multicolumn{3}{|c|}{ Chest Discomfort } \\
\hline & \multicolumn{2}{|c|}{ Present } & Absent \\
\hline & $\overline{\mathbf{N}}$ & $\%$ & \\
\hline With shortness of breath (SOB) & 3 & 25.0 & \\
\hline Without SOB & 7 & 58.3 & \\
\hline Total & 10 & & 2 \\
\hline
\end{tabular}

A total of $10(83.3 \%)$ patients had perfusion defect in LAD territory, $8(66.7 \%)$ in LCX territory and $8(66.7 \%)$ in RCA territory. 3(25.0\%) had fixed defect, 5(41.7\%) patients had partial reversible defect and 4(33.3\%) patients had complete reversible defect in pre intervention MPI (Table-4a and $4 \mathrm{~b}$ ). In pre-intervention
MPI the sensitivity, specificity and positive predictive value of detecting perfusion defect in case of LAD territory are $90.0 \%, 100 \%$ and $100 \%$ respectively. In case of LCX territory they are $77.8 \%, 66.7 \%$ and $87.5 \%$ respectively. In case of RCA territory the sensitivity, specificity and positive predictive value are $81.8 \%$, $100 \%$ and $100 \%$ respectively (Table-5).

Table 4 (a) : Distribution of the study subjects on the basis of involved coronary vascular territory in pre intervention MPI

\begin{tabular}{lll}
\hline $\begin{array}{l}\text { Vascular Territory of } \\
\text { Perfusion defect }\end{array}$ & Number & Percentage \\
LAD territory & 10 & 83.3 \\
LCX territory & 8 & 66.7 \\
RCA territory & 8 & 66.7 \\
\hline
\end{tabular}

Table 4 (b) : Distribution of the study subjects on the basis of type of perfusion defect in pre intervention MPI

\begin{tabular}{lll}
\hline Type of perfusion defect & Number & Percentage \\
\hline Fixed defect & 3 & 25 \\
Partial reversible defect & 5 & 41.7 \\
Complete reversible defect & 4 & 33.3 \\
\hline
\end{tabular}

Table 5: Validity test of detecting perfusion defect in pre-intervention MPI compared to CAG.

\begin{tabular}{|c|c|c|c|c|c|}
\hline $\begin{array}{l}\text { Vascular } \\
\text { territories } \\
\text { having } \\
\text { perfusion } \\
\text { defect } \\
\end{array}$ & $\begin{array}{c}\text { Sensitivity } \\
(\%)\end{array}$ & $\begin{array}{c}\text { Specificity } \\
(\%)\end{array}$ & $\begin{array}{c}\text { Accuracy } \\
(\%)\end{array}$ & $\begin{array}{c}\text { Positive } \\
\text { predictive } \\
\text { value } \\
(\%)\end{array}$ & $\begin{array}{c}\text { Negative } \\
\text { predictive } \\
\text { value } \\
(\%)\end{array}$ \\
\hline $\begin{array}{l}\text { LAD in pre- } \\
\text { intervention } \\
\text { MPI }\end{array}$ & 90.0 & 100.0 & 91.7 & 100.0 & 66.7 \\
\hline $\begin{array}{l}\text { LCX in pre- } \\
\text { intervention } \\
\text { MPI }\end{array}$ & 77.8 & 66.7 & 75.0 & 87.5 & 50.0 \\
\hline $\begin{array}{l}\text { RCA in pre- } \\
\text { intervention } \\
\text { MPI }\end{array}$ & 81.8 & 100.0 & 83.3 & 100.0 & 33.3 \\
\hline
\end{tabular}

Table 6 summarizes the distribution of study patients by intervention. In post intervention MPI it was observed that $5(41.6 \%)$ patients had perfusion defect in LAD territory, 6(50\%) in LCX territory and 5(41.6\%) in RCA territory, 4(33.3\%) patients had partially reversible perfusion defect, $2(16.7 \%)$ patients had fixed perfusion 
defect, 1(3.3\%) patient had completely reversible perfusion defect and 5(41.7) patients had normal MPI findings (Table-7). Table 8 shows the comparison between pre intervention and post intervention MPI findings of perfusion defects.

Table 6: Distribution of the study subjects by intervention $(n=12)$

\begin{tabular}{cccc}
\hline \multirow{2}{*}{ Coronary vascular territory } & \multicolumn{3}{c}{ Coronary intervention } \\
\cline { 2 - 4 } & PCI & CABG & PCI+CABG \\
\hline LAD & 3 & 1 & 1 \\
LCX & 5 & 1 & 0 \\
RCA & 3 & 0 & 0
\end{tabular}

Table 7: Distribution of the study subjects by involved vascular territory and type of perfusion defect in post intervention MPI study ( $n=12)$

\begin{tabular}{lcc}
\hline $\begin{array}{l}\text { Vascular territory of } \\
\text { Perfusion defect }\end{array}$ & Number & Percentage \\
\hline LAD territory & 5 & 41.6 \\
LCX territory & 6 & 50 \\
RCA territory & 5 & 41.6 \\
Type of perfusion defect & & \\
$\quad$ Fixcd perfusion defcct & 2 & 16.7 \\
Partial revcrible pcrfusion defect & 4 & 33.3 \\
Complctc revcrsible perfusion defect & 1 & 8.3 \\
$\quad$ Normal & 5 & 41.7 \\
\hline
\end{tabular}

Table 8: Comparisonbetween pre intervention and post intervention MPI findings of perfusion defects $(n=12)$

\begin{tabular}{|c|c|c|c|c|c|c|c|}
\hline \multicolumn{3}{|c|}{ Pre intervention MPI } & \multicolumn{4}{|c|}{ Post intervention MPI } & \multirow[t]{3}{*}{$P$ value } \\
\hline & & & \multicolumn{2}{|c|}{$\begin{array}{r}\text { Perfusion } \\
\text { defect present }\end{array}$} & \multicolumn{2}{|c|}{ Normal } & \\
\hline & $\mathbf{N}$ & $\%$ & $\mathbf{N}$ & $\%$ & $\mathbf{N}$ & $\%$ & \\
\hline Fixed defect & 3 & 25.0 & 2 & 16.7 & 1 & 20.0 & $>0.05$ \\
\hline Partial reversibility & 5 & 41.7 & 4 & 33.3 & 1 & 20.0 & $>0.05$ \\
\hline Complete reversibility & 4 & 33.3 & 1 & 8.3 & 3 & 60.0 & $>0.05$ \\
\hline
\end{tabular}

Table 9: Comparison between pre and post intervention MPI findings on the basis of involved coronary vascular territory $(n=12)$

\begin{tabular}{|c|c|c|c|c|c|c|c|c|c|c|c|}
\hline \multirow{3}{*}{$\begin{array}{l}\text { Coronary } \\
\text { vascular } \\
\text { territory }\end{array}$} & \multicolumn{5}{|c|}{ Pre-intervention YPI } & \multicolumn{5}{|c|}{ Posi-intervention MPI } & \multirow[t]{3}{*}{ P-ralue } \\
\hline & \multirow{2}{*}{$\begin{array}{l}\text { No. of } \\
\text { perfusion } \\
\text { defect }\end{array}$} & \multicolumn{4}{|c|}{ Type of perfusion defect } & \multirow{2}{*}{$\begin{array}{l}\text { Yo. of } \\
\text { perfusion } \\
\text { defect }\end{array}$} & \multicolumn{3}{|c|}{ Type of perfusion defect } & & \\
\hline & & Fixed & $\begin{array}{l}\text { Comple } \\
\text { reversil }\end{array}$ & $\begin{array}{l}\text { artial } \\
\text { eversible }\end{array}$ & Mixed & & & $\begin{array}{l}\text { Compl } \\
\text { reversil }\end{array}$ & $\begin{array}{l}\text { artial } \\
\text { versible }\end{array}$ & Nixed & \\
\hline LAD & $\begin{array}{c}10 \\
(27.7 \%)\end{array}$ & 3 & 3 & 3 & 1 & $\begin{array}{c}5 \\
(13.8 \%)\end{array}$ & 3 & 1 & 0 & 1 & $>0.05$ \\
\hline LCX & $\begin{array}{c}8 \\
(22.2 \%)\end{array}$ & 2 & 3 & 2 & 1 & $\begin{array}{c}6 \\
(16.6 \%)\end{array}$ & 3 & 1 & 0 & 2 & \\
\hline RCA & $\begin{array}{c}8 \\
(22.2 \%)\end{array}$ & 1 & 5 & 1 & 1 & $\begin{array}{c}5 \\
(13.8 \%)\end{array}$ & 3 & 0 & 1 & 1 & \\
\hline
\end{tabular}

Table 10: Comparison of percentage of involvement of myocardium by the fixed defects in preintervention MPI with post intervention MPI on the basis of involved vascular territory and total left ventricular myocardium $(n=12)$

Percentage of Involvement of Myocardium
Pre Intervention Post Intervention P value

Mean \pm SE Range Mean \pm SE Range

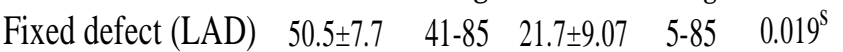
Fixed defect (LCX) $\quad 20.08 \pm 5.5 \quad 10-50 \quad 10.42 \pm 3.6 \quad 3-26 \quad 0.160^{\text {ns }}$ Fixed defect (RCA) $31.75 \pm 10.8 \quad 5-95 \quad 9.0 \pm 4.1 \quad 8-45 \quad 0.104^{\text {ns }}$ $\begin{array}{llllll}\text { Fixed defect } \quad 44.45 \pm 4.6 & 18-70 & 19.73 \pm 6.9 & 5-53 & 0.019^{\mathrm{S}}\end{array}$ (total LV myocardium)

Table 11: Comparison of preintervention and post intervention MPI findings on the basis of Summed Stress Score (SSS) $(n=12)$

\begin{tabular}{ccccc}
\hline \multicolumn{4}{c}{ Pre Intervention } & Post Intervention \\
\hline $\mathrm{N}$ & $\%$ & $\mathrm{~N}$ & $\%$ \\
\hline
\end{tabular}

\begin{tabular}{lccccc} 
Mild risk $(<3-8)$ & 0 & 0.0 & 1 & 8.3 & $0.697^{\text {ns }}$ \\
Moderate risk $(9-13)$ & 3 & 25.0 & 2 & 16.7 & \\
High risk $(>13)$ & 4 & 33.3 & 3 & 25.0 & \\
\hline
\end{tabular}

$\mathrm{p}$ value reached from Chi-square test

Table 12: Comparison of the study subjects by pre and post intervention MPI finding on the basis of LVEF (\%) (n=12)

\begin{tabular}{|c|c|c|c|c|c|}
\hline & \multicolumn{2}{|c|}{ Pre Intervention } & \multicolumn{2}{|c|}{ Post Intervention } & \multirow{2}{*}{ P value } \\
\hline & Mean & $\pm \mathrm{SD}$ & Mean & $\pm \mathrm{SD}$ & \\
\hline MPI- LVEF stress & 50.6 & \pm 13.2 & 57.1 & \pm 15.9 & $0.082^{\mathrm{ns}}$ \\
\hline Range (min-max) & 26 & .65 & 35 &, 72 & \\
\hline MPI-LVEF Rest & 47.7 & \pm 11.2 & 55.0 & \pm 15.1 & $0.077^{\mathrm{ns}}$ \\
\hline Range (min-max) & 29 & ,65 & 33 &, 72 & \\
\hline
\end{tabular}


Table 13: Comparison between pre and post intervention MPI SPECT gating findings $(n=12)$

\begin{tabular}{lccccc}
\hline & \multicolumn{6}{c}{ Pre Intervention } & \multicolumn{5}{c}{ Post Intervention P value } \\
& N & $\%$ & n & $\%$ & \\
\hline MPI- Wall motion & & & & & \\
Normal & 6 & 50.0 & 7 & 58.3 & \\
Abnormal & 6 & 50.0 & 5 & 41.7 & $1.000^{\mathrm{ns}}$ \\
MPI- Wall Thickening & & & & & \\
Normal & 8 & 66.7 & 8 & 66.7 & $1.000^{\mathrm{ns}}$ \\
Wall thinning & 4 & 33.3 & 4 & 33.3 & $1.000^{\mathrm{ns}}$ \\
MPI- LV cavity & & & & & \\
Normal & 8 & 66.7 & 8 & 66.7 & \\
Borderline Dilated & 2 & 16.7 & 3 & 25.0 & $1.000^{\mathrm{ns}}$ \\
Dilated & 2 & 16.7 & 1 & 8.3 & \\
MPI- TID & & & & & \\
Normal & 9 & 75.0 & 8 & 66.7 & \\
Higher & 3 & 25.0 & 4 & 33.3 & $0.653^{\mathrm{ns}}$ \\
MPI-Cardiomyopathy & & & & & \\
Absent & 8 & 66.7 & 8 & 66.7 & \\
Present & 4 & 33.3 & 4 & 33.3 & $1.000^{\mathrm{ns}}$ \\
\hline p vale reas & & & &
\end{tabular}

$\mathrm{p}$ value reached from McNemar test

Table- 14: Distribution of the study subjects by history of restenosis in CAG and Post-intervention MPI (n=12)

\begin{tabular}{lccccc}
\hline $\begin{array}{l}\text { History of Number of Percentage } \\
\text { restenosis in } \\
\text { patients }\end{array}$ & $\begin{array}{c}\text { History of } \\
\text { restenosis in }\end{array}$ & $\begin{array}{c}\text { Number of } \\
\text { patients }\end{array}$ & Percentage \\
CAG & & & MPI & & \\
\hline Present & 4 & 33.33 & Present & 2 & 16.67 \\
Absent & 8 & 66.67 & Absent & 10 & 83.33 \\
\hline
\end{tabular}

\section{DISCUSSION}

At the beginning 22 patients were enrolled according to selection criteria. 2 patients died during the study period before undergoing post-intervention MPI, 4 patients did not undergo coronary intervention even after being advised by their respective physicians and 4 patients did not come for follow up MPI after intervention within the study period. After excluding these 10 patients we completed the study with 12 CAD patients. The mean age of study patients was $55.58 \pm$ 11.14 (mean $\pm \mathrm{SD}$ ) years of whom $9(75 \%)$ were male.

The result of pre-intervention MPI revealed the perfusion defect was mostly found in LAD territory which was about $83.3 \%$. It was also observed in the study of LeBlanc et al.
2016, where LAD territory was involved in $60 \%$ of STEMI patients and $96 \%$ of chronic stable angina patients

(1).The sensitivity of pre intervention MPI for the initial evaluation and risk stratification by diagnosing perfusion defect in these patients have been found to be $90 \%, 77.8 \%$ and $81.8 \%$ in case of LAD, LCX and RCA territories respectively. Whereas the specificity and positive predictive value have been found $100 \%$ for LAD and RCA territories compared to Coronary Angiography being the gold standard (Table-5). The overall performance of SPECT-MPI for the detection of myocardial ischemia was $79 \%$ for both sensitivity and specificity in a study done by Galassi et al. 2011 (2). The meta-analysis of Takx et al. shows that sensitivity and specificity of SPECT MPI is $74 \%$ and $79 \%$ respectively (3). In the present study, the sensitivity, specificity and positive predictive value were found higher as the pre intervention MPI was performed within 3-4 months after coronary angiography in most of the patients. The use of stress nuclear imaging increases significantly the sensitivity of detecting perfusion defect to $87 \%$ and the specificity to $78 \%$ (4). So, it is more favorable to take the coronary intervention decisions considering the MPI results as it can predict the perfusion defects more specifically. Present study shows similar results establishing SPECT MPI as the most reliable non invasive method in diagnosing significant perfusion defect in known or suspected CAD patients and whether they can be improved by revascularization or not.

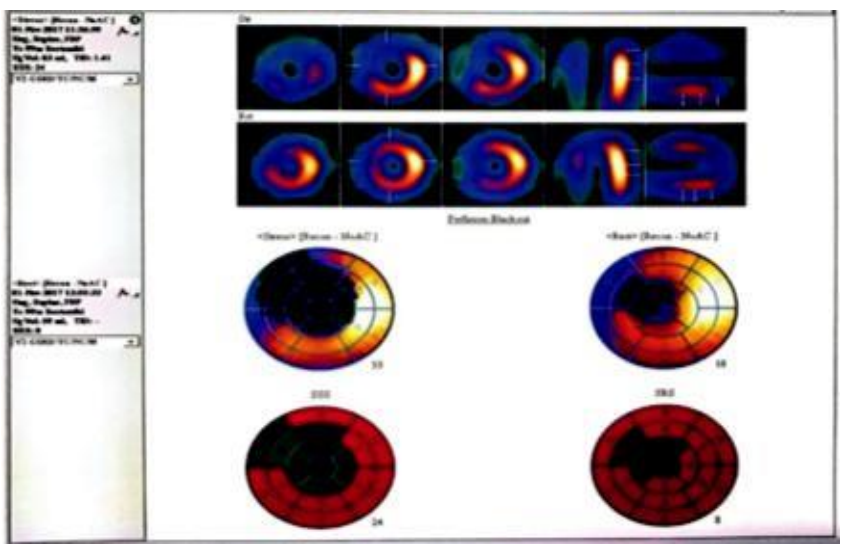

Figure 2: The photograph of pre intervention MPI of a 46 years old male CAD patient showing large fixed perfusion defect with large area of peri-infarct ischemia involving LAD territory and reversible perfusion defect in LCX and RCA territories representing ischemia 

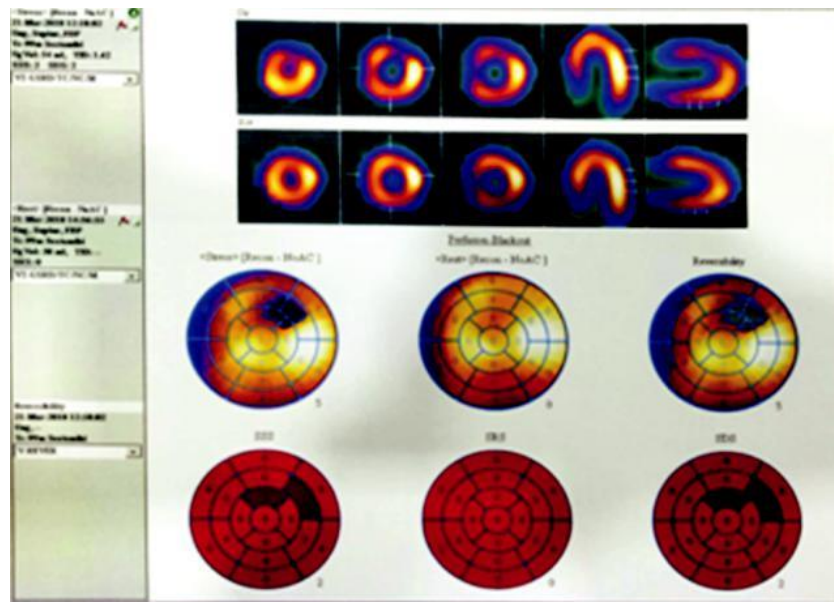

Figure 3: The photograph of post-intervention MPI of the same patient after 3 months of undergoing PTCA with stenting to LAD showing very small reversible perfusion defect in $L A D \&$ LCX territories represent ischemia.

Approximately $60 \%$ of patients with completely reversible perfusion defect in pre-intervention MPI had normal findings in follow up MPI done after intervention and 20\% patients having partially reversible perfusion defects in pre-intervention MPI had normal finding in follow up MPI (Table-8). SPECT myocardial perfusion imaging provided significant prognostic information for the prediction of outcome in these patients. In a previous study done on known or suspected CAD patients undergoing MPI in 2012 about $08 \%$ patients had completely reversible perfusion defects, $12 \%$ had partially reversible perfusion defects and $30 \%$ had fixed perfusion defects before undergoing intervention. During a follow up of $9.2 \pm 2$ years it was observed that patients with an abnormal SPECT study had a significantly increased MACE (Major Advance Cardiac Events) rate as compared with patients who had normal MPI results previously (5). Before intervention the mean percentage of involved myocardium by fixed defect in LAD territory was $50.5 \pm 7.7$ with ranged from $41 \%$ to $85 \%$ and after intervention the mean percentage was 21.7

\pm 9.07 with ranged from $05 \%$ to $85 \%$ (Table-10). In case of total LV myocardial involvement the mean percentage of fixed defect also significantly reduced in post intervention MPI than the pre intervention MPI. These two results are statistically significant which depicts the positive outcome of coronary intervention on fixed defect.

Risk stratification using myocardial perfusion SPECT Summed Stress Score (SSS) has been proved to be crucial by providing incremental information over clinical data for prediction of outcome. SSS is considered to be an independent predictor of MACE (Major Adverse Cardiac Events) (6). It is obtained by adding the scores of the 20 segments of the stress Tc99m Sestamibi SPECT images. A summed stress score of $<4$ is considered normal, $4-8$ is mildly abnormal and $>8$, severely abnormal.6 In this study it was observed that $33.3 \%$ patients were in high risk before undergoing intervention, whereas it was found to be $25 \%$ after intervention in these patients (Table-11).

Among total 12 patients, 04 patients had undergone CAG after intervention and found to have developed restenosis. 02(16.67\%) patients had perfusion defect in the stented vascular territory in post intervention MPI which can be regarded as restenosis (Table-14). That helped the physicians in making further management plan in these patients i.e whether to go for coronary intervention again or not. The incidence of development of restenosis was found to be $31 \%$ in a study by Galassi et al. 2000 where SPECT MPI was performed after 5-6 months of coronary intervention (7). Post intervention MPI has been performed mostly after 3-4 months in current study which may have influenced the lower percentage of re-stenosis compared to the previous study. Also, if we could include the post intervention CAG result in all the study subjects then we could have better scenario of sensitivity of detecting restenosis in post intervention MPI in present study.

SPECT MPI has disadvantages as radiation exposure to the patient and higher costs. Radiation exposure is an increasingly relevant topic as myocardial perfusion SPECT studies are an important source of medical radiation exposure and because patients frequently have multiple procedures performed over time. It is said that new cadmium zinc telluride SPECT camera technology may substantially reduce radiation exposure while 
maintaining image quality (8). Moreover, an individualized imaging protocol may further reduce both study time and radiation exposure. If the patients have normal stress myocardial perfusion images, then in these patients resting images are not necessary and a further reduction in the radiation dose can be achieved by using a stress-only imaging protocol (9).Cost is another factor limiting routine risk stratification using myocardial perfusion SPECT when compared with other non-invasive methods i.e exercise electrocardiographic testing. But suboptimal accuracy of exercise electrocardiographic testing may result in potential misdiagnosis of obstructive coronary artery disease, which may hinder appropriate patient care. Additionally, myocardial perfusion SPECT has a high negative predicting value, which may avoid the use of unnecessary (invasive) diagnostic procedures (9). In previous studies it has been found that the costs were significantly lower after using SPECT MPI rather than CTA or PET in the evaluation of suspected coronary disease. SPECT has been found to be economically attractive compared with PET, whereas CTA was associated with higher costs and also there was no significant difference in mortality compared with SPECT (10).

\section{CONCLUSION}

The results of this study have indicated that SPECT MPI provides significant independent information concerning the outcome of coronary intervention in CAD patients. The sensitivity of predicting the outcome of post coronary intervention is $>75 \%$ in all 3 major coronary vascular territories, whereas the specificity and PPV both are $100 \%$ in 2 out of 3 major vascular territories. Furthermore, early SPECT MPI after intervention successfully identified significant improvement of myocardial viability after revascularization in patients having complete or reversible perfusion defects found in pre intervention MPI along with identifying those having restenosis. It could also depict the reduction of percentage of myocardial fixed defects within 3-4 months after intervention which is also a positive outcome of coronary revascularization.

\section{RECOMMENDATION}

Future studies with large number of study subjects should be performed for better specification of this result.

\section{REFERENCES}

1. LeBlanc S, Bibeau K, Bertrand OF, Lévesque V, Deschênes StPierre B, Pibarot P, Després JP, Larose E. Carotid versus coronary atherosclerosis burdens in acute compared with chronic symptomatic coronary artery disease. Canadian journal of physiology and pharmacology. 2017 May 18;95(8):878-87.

2. Galassi AR, Marzá F, Azzarelli S, Tomasello SD. Role of stress myocardial scintigraphy in the evaluation of incompletely revascularized post-PCI patients. International journal of molecular imaging. 2011;2011.

3. Takx RA, Blomberg BA, Aidi HE, Habets J, de Jong PA, Nagel E, Hoffmann U, Leiner T. Diagnostic accuracy of stress myocardial perfusion imaging compared to invasive coronary angiography with fractional flow reserve meta-analysis. Circulation: Cardiovascular Imaging. 2015 Jan;8(1):e002666.

4. Dori G, Denekamp Y, Fishman S, Bitterman H. Exercise stress testing, myocardial perfusion imaging and stress echocardiography for detecting restenosis after successful percutaneous transluminal coronary angioplasty: a review of performance. Journal of internal medicine. 2003 Mar;253(3):253-62.

5. Schinkel AF, Boiten HJ, Van Der Sijde JN, Ruitinga PR, Sijbrands EJ, Valkema R, Van Domburg RT. Prediction of 9-year cardiovascular outcomes by myocardial perfusion imaging in patients with normal exercise electrocardiographic testing. European Heart JournalCardiovascular Imaging. 2012 May 15;13(11):900-4.

6. Galassi AR, Grasso C, Azzarelli S, Ussia G, Moshiri S, Tamburino C. Usefulness of exercise myocardial scintigraphy in multivessel coronary disease after incomplete revascularization with coronary stenting. The American journal of cardiology. 2006 Jan 15;97(2):207-15.

7. Galassi AR. Usefulness of exercise tomographic myocardial perfusion imaging for detection of restenosis after coronary stent implantation. Am J Cardiol. 2000;85:1362-4.

8. Sharir T, Slomka PJ, Hayes SW, DiCarli MF, Ziffer JA, Martin WH, Dickman D, Ben-Haim S, Berman DS. Multicenter trial of high-speed versus conventional single-photon emission computed tomography imaging: quantitative results of myocardial perfusion and left ventricular function. Journal of the American College of Cardiology. 2010 May 4;55(18):1965-74.

9. Chang SM, Nabi F, Xu J, Raza U, Mahmarian JJ. Normal stressonly versus standard stress/rest myocardial perfusion imaging: similar patient mortality with reduced radiation exposure. Journal of the American College of Cardiology. 2010 Jan 19;55(3):221-30.

10. Hlatky MA, Shilane D, Hachamovitch R, DiCarli MF, SPARC Investigators. Economic outcomes in the study of myocardial perfusion and coronary anatomy imaging roles in coronary artery disease registry: the SPARC study. Journal of the American College of Cardiology. 2014 Mar 18;63(10):1002-8. 